\title{
Atrial Infarct: An Easily Missed Reality with Hidden Threat - A case report with Review of Literature
}

\author{
Mohammed Abaye Deen Saleh ${ }^{1}$, Abdul Wadud Chowdhury ${ }^{1}$, Pratyay Hasan ${ }^{2}$, Syed Rezwan \\ Kabir ${ }^{1}$, Khyrun Nahar ${ }^{1}$, KMN Sabah ${ }^{1}$, Mohammad Gaffar Amin ${ }^{1}$, Kazi Nazrul Islam ${ }^{1}$ \\ ${ }^{1}$ Department of Cardiology, Dhaka Medical College Hospital, Dhaka, ${ }^{2}$ Upazilla Health Complex, \\ Dhamrai, Dhaka
}

Key Words : Ischaemic heart disease, atrium.

\begin{abstract}
Atrial infarction is a very rare diagnosis. Though available literature suggests, the condition per se may have been not so rare. Over the past few decades, atrial infarction have been reported several times, even some case series have been reported, but there is no consensus on the diagnosis of this condition, and its true importance also has not been understood completely. Previous works have shown that this condition is associated with several serious complications; hence recognition of this condition in early period is important, which is at the same time not so easy due to subtlety of the known features and less availability of information. We report a case of 70 year old Muslim, Bengali, male suffering from acute coronary syndrome, in whom, right atrial infarction was recognized by electrographic features, which is very rarely diagnosed with confidence in ante-mortem patients.

Since, in Bangladesh, post-mortem autopsy to find out causes behind cardiac death is not done routinely and in the light of possibility of serious life-threatening complications, ante-mortem diagnosis of atrial infarction is necessary. So, Cardiologists should be aware of this uncommon condition.
\end{abstract}

(Cardiovasc. j. 2015; 8(1): 65-68)

\section{Introduction:}

Atrial Infarction is among the least encountered diagnoses in clinical cardiology, albeit having a much higher true incidence $(1-42 \%)$ as evidenced by the autopsy. ${ }^{1-3}$ The reason behind these are absence of distinctive clinical presentation, agreed ECG criteria for diagnosis, and small, inconspicuous nature of the ECG changes. ${ }^{2-5}$ Although a few cases have been reported over the span of almost half of the past century and in recent years, amount of available literature, especially amount of case reports is truly scanty on this potentially life-threatening condition and lesser is the information available in the textbooks. ${ }^{1,6,7} \mathrm{We}$ intend to report a case of right atrial infarction who presented in Coronary Care Unit of Dhaka Medical College Hospital, Dhaka, Bangladesh.

\section{Case Report:}

A 70 year old male got admitted into Dhaka Medical College Hospital within two hours of onset of severe ischaemic type of chest pain and with ECG features of ST elevated acute inferior myocardial infarction with right ventricular infarct with right atrial infarct with episodes of atrial ectopic. He was aggressively treated with fibrinolytic (streptokinase) and other necessary management for acute ST elevated inferior MI with right ventricular infarct with right atrial infarct. Following features suggestive of right atrial infarction, were recognized in the ECG of our patient (see Figure 1):

1. ST segment elevation in the inferior leads

2. $\mathrm{PR}$ segment elevation in aVR and V1

3. PR segment depression in leads II, III and aVF, and

4. $\mathrm{P}$ wave axis $83^{0}$

Echocardiography done on the next day showed mild hypokinesia of inferior wall with trivial mitral

Address of Correspondence: Dr. Mohammed Abaye Deen Saleh, Department of Cardiology, Dhaka Medical College Hospital, Dhaka, Bangladesh. Email: sarahcosis@gmail.com 


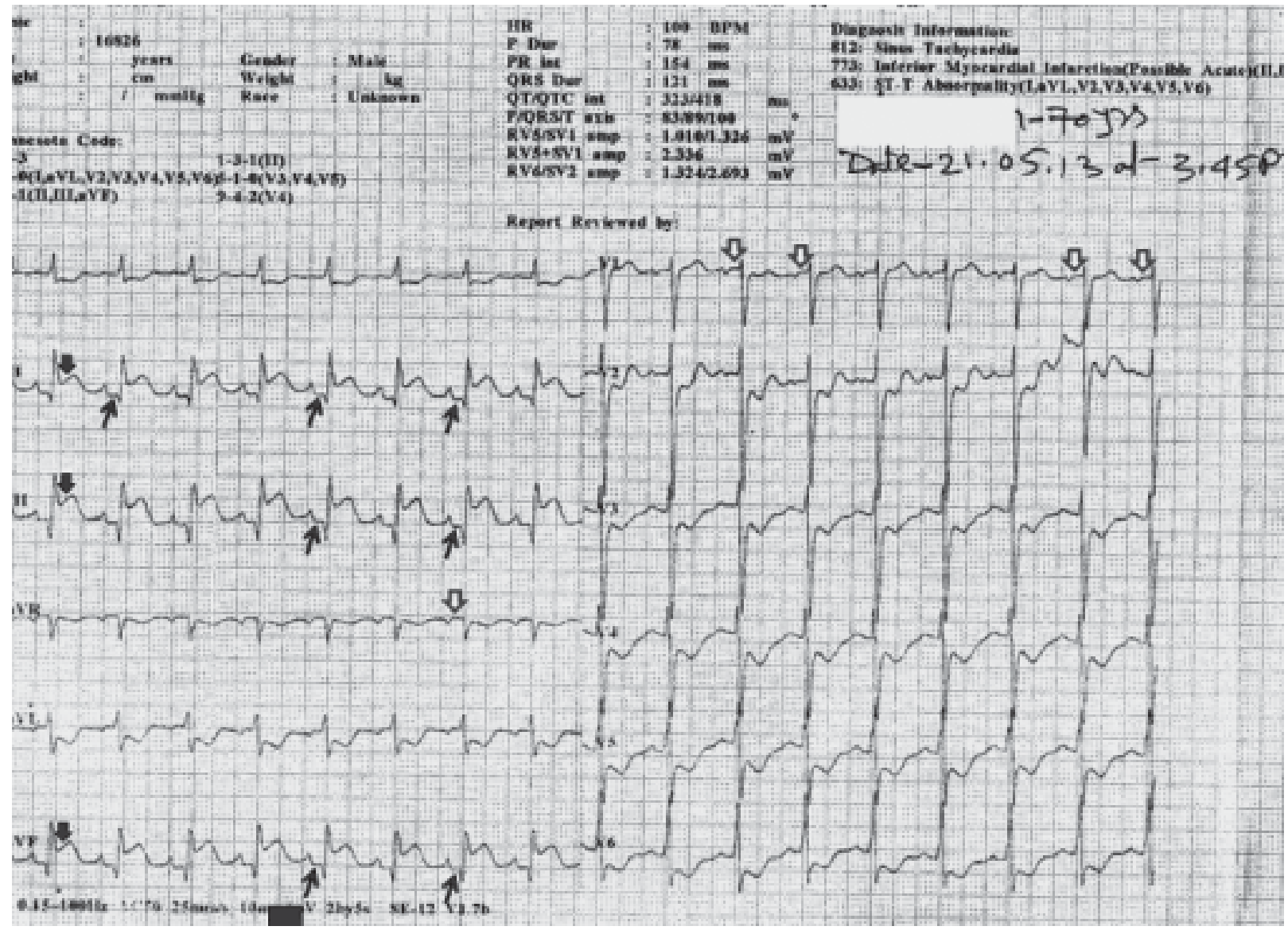

Fig.-1: Showing ECG changes found in the patient (ST segment elevation in the inferior leads (II, III. $a V F), P R$ segment elevation in $a V R$ and V1, PR segment depression in leads II, III, and aVF, and P wave axis was $83^{\circ}$ ).

regurgitation with good left ventricular systolic function (LVEF 66\%) which indicated that patient responded well to the initial treatment. The patient was discharged after five days with advice and follow-up appointments scheduled for the outpatient clinic. Angiogram of the patient was done after one and half month and stenosis (80\%) of the proximal right coronary artery was detected. Percutaneous coronary intervention was done with drug eluting stent (DES) with good results.

\section{Discussion:}

As mentioned earlier, there is no consensus yet reached on the criteria for diagnosing atrial infarction. In recent years, Shakir et.al. diagnosed right atrial infarction based on the following characteristics $^{6}$ :

1. ST segment elevation in the inferior leads

2. $\mathrm{PR}$ segment elevation in $\mathrm{aVR}$ and $\mathrm{V} 1$
3. PR segment depression in leads II, III and aVF, and

4. $\mathrm{P}$ wave axis deviation

Another criteria suggested by Sivertssen et. al. are as follows ${ }^{8}$ :

1. P-R prolongation (normal $<200 \mathrm{~ms}$ )

2. $\mathrm{P}$ wave axis changes (normal axis $\mathrm{P}$ wave in frontal plane lies between $30^{\circ}-60^{\circ}$.)

3. Abnormal atrial rhythm including atrial flutter, atrial fibrillation, wandering pacemaker and AV nodal rhythm.

Our case had ECG findings identical to each of those characteristics, described by Shakir et. al. as significant ST segment elevation was found (2.5$4 \mathrm{~mm}$ in inferior leads II, III, aVF). ${ }^{6}$ Axis deviation was present $\left(83^{\circ}\right)$, additional criterion criterion for diagnosing atrial infarction by Sivertssen et. al. 
and as well as abnormal atrial rhythm (atrial ectopic) which was also seen in our patient. ${ }^{8}$ Thus our case matches all of the criteria reported by Shakir et. al. and two out of three criteria proposed by Sivertssen et. Al. ${ }^{6,8}$ Liu et.al. ${ }^{5}$ suggested to maintain high degree of suspicion of atrial infarction in patients having any form of atrial dysrhythmia.

They also proposed the following criteria for diagnosing atrial infarction:

1. Major criteria

a. Elevation of P-Ta segment $>0.5 \mathrm{~mm}$ in lead $\mathrm{V}_{5} \& \mathrm{~V}_{6}$ with reciprocal changes seen in lead $\mathrm{V}_{1}$ and $\mathrm{V}_{2}$.

b. Elevation of P-Ta segment $>0.5 \mathrm{~mm}$ in lead I with reciprocal changes seen in lead II or III.

c. Depression of P-Ta segment $>1.5 \mathrm{~mm}$ in precordial leads and depression $>1.2 \mathrm{~mm}$ in leads I, II, III in the presence of any form of atrial arrhythmia.

2. Minor criteria:

a. Abnormal P wave: M/W/irregular/notched

Depression of P-Ta segment of small amplitude in absence of elevation in the other leads cannot be regarded itself as a positive evidence of atrial infarction. Diagnosis of atrial infarction cannot be made when ventricular infarction cannot be diagnosed.

Usual causes of primary PR segment changes include ${ }^{6,9}$ :

1. Pericarditis

2. Pericardial effusion

3. Atrial infarction

4. Atrial injury due to penetrating trauma.

In more recent years, Jim Man-Hong et. al. found a profound $\mathrm{P}-\mathrm{R}$ depression of $\geq 1.2 \mathrm{~mm}$ in inferior leads was associated with a complicated hospital course and poor short term outcome in acute inferior MI. ${ }^{9}$ They also found that in most of the MI patients with pericardial effusion (64.8\%), the $\mathrm{P}-\mathrm{R}$ segment depression was $<0.5 \mathrm{~mm}$, on the other hand, there was no pericardial effusion in half of patients with P-R depression $>1.2 \mathrm{~mm}$. Gardin et. al. commented that there is relatively higher incidence of right atrial infarct (81-98\%) compared to left atrial infarct. ${ }^{1}$
Atrial infarction is associated with potential complications like-

1. Supraventricular tachycardia (61\%). ${ }^{10}$

2. Atrial rupture (4.5\%). ${ }^{10}$

3. Intra-atrial thrombosis $(80-84 \%)^{2,11}$

4. Pulmonary / peripheral embolism (24\%). ${ }^{12}$

Possibility of serious life-threatening complications such as those mentioned above necessitates antemortem diagnosis of atrial infarction and accurate recognition of the hidden occurrence of this condition.

\section{Conclusion:}

In our case prompt diagnosis of ST elevated inferior MI with right ventricular infarct with right atrial infarct with atrial ectopic was possible and management was done aggressively, to which the patient responded surprisingly well. So to diagnose this condition in susceptible patients, special attention is needed. In Bangladesh, where postmortem autopsy is almost never performed as a routine practice, ante-mortem diagnosis is all the more necessary. Though few cases have been reported, potentially life threatening complications have been documented in most of them indicating seriousness of the condition and importance of diagnosis. We recommend further research directed at developing specific and easily implementable diagnostic criteria for diagnosing atrial infarction.

\section{Conflict of Interest - None.}

\section{References:}

1. Gardin JM, Singer DH. Atrial infarction. Importance, diagnosis, and localization. Arch Intern Med 1981 Sep;141(10):1345-1348.

2. Cushing EH, Feil HS, Stanton EJ, Wartman WB. Infarction of the cardiac auricles (atria): clinical, pathological, and experimental studies. Br Heart $J 1942$ Jan;4(1-2):17-34.

3. McCain FH, Kline EM, Gilson JS. A clinical study of 281 autopsy reports on patients with myocardial infarction. Am Heart J 1957;39(2):263-272.

4. Lazar EJ, Goldberger J, Peled H, Sherman M, Frishman WH. Atrial infarction: Diagnosis and management. Am Heart J 1988 Oct;116(4):1058-1063. 
5. Liu CK, Greenspan G, Piccirillo RT. Atrial infarction of the heart. Circulation 1961 Mar;23:331-338.

6. Shakir DK, Arafa SO. Right atrial infarction, atrial arrhythmia and inferior myocardial infarction form a missed triad: A case report and review of the literature. Can J Cardiol 2007 Oct;23(12):995-997.

7. Mendes RG, Evora PR. Atrial infarction is a unique and often unrecognized clinical entity. Arq Bras Cardiol 1999 Mar;72(3):333-342.

8. Sivertssen E, Hoel B, Bay G, Jörgensen L. Electrocardiographic atrial complex and acute atrial myocardial infarction. Am J Cardiol 1973 Apr;31(4): $450-456$.
9. Jim M-H, Siu C-W, Chan AO-O, Chan RH-W, Lee SWL, Lau C-P. Prognostic implications of PR-segment depression in inferior leads in acute inferior myocardial infarction. Clin Cardiol 2006 Aug 1;29(8):363-368.

10. Söderström N. Myocardial infarction and mural thrombosis in the atria of the heart. Acta Med Scand Suppl 1948;217:1-114.

11. Wartman WB, Hellerstein HK. The incidence of heart disease in 2,000 consecutive autopsies. Ann Intern Med 1948 Jan;28(1):41-65.

12. Hellerstein HK. Atrial infarction with diagnostic electrocardiographic findings. Am Heart J 1948 Sep;36(3):422-430. 